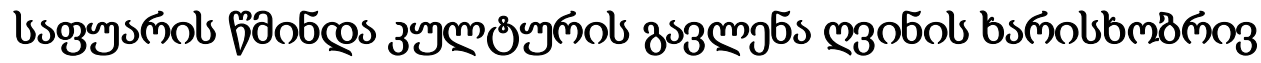

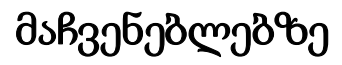

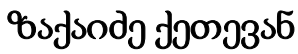

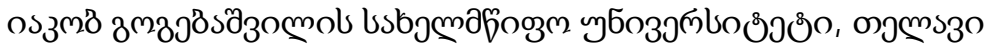

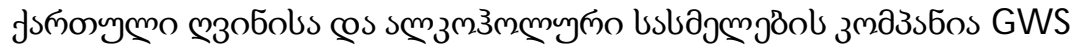 \\ uscoos amjaj

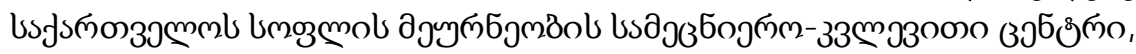

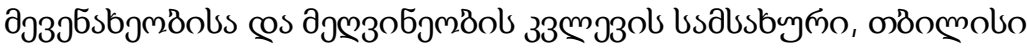

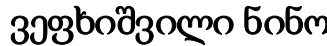

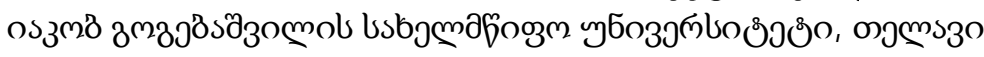

https://doi.org/10.52340/idw.2021.494

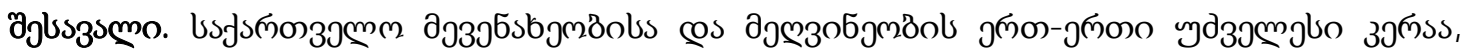

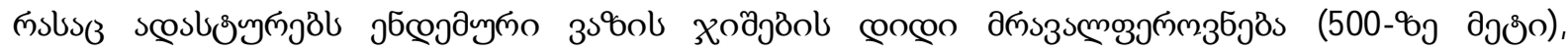

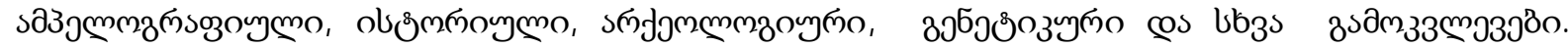

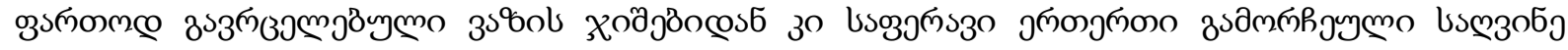

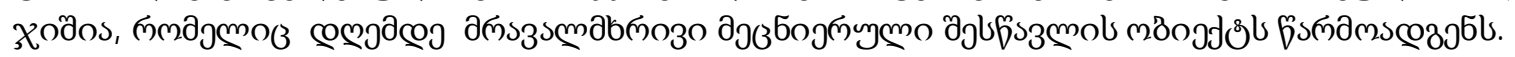

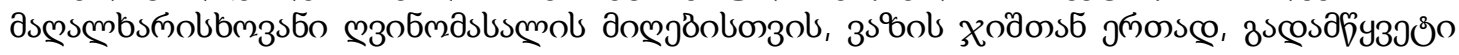

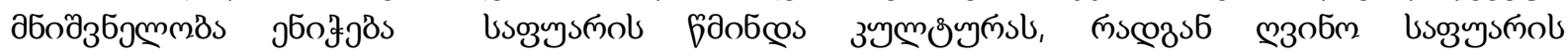

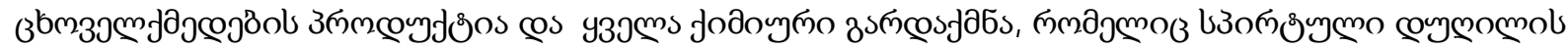

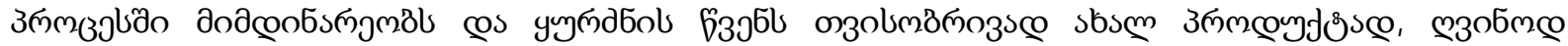

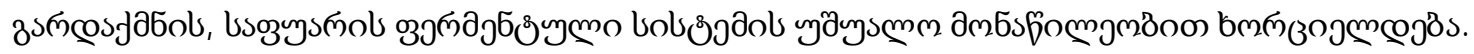

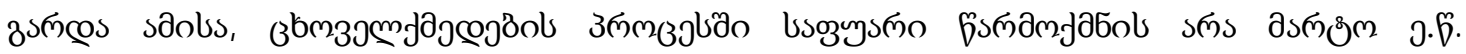

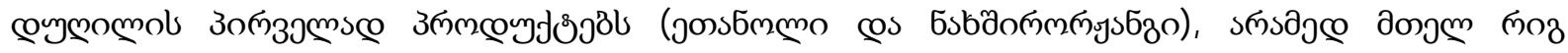

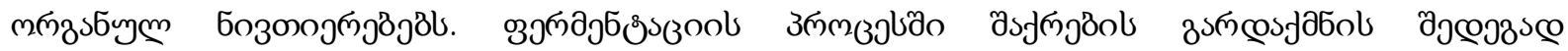

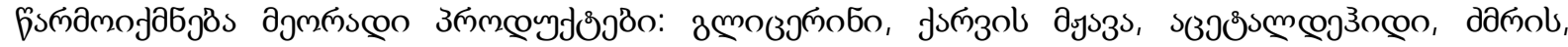

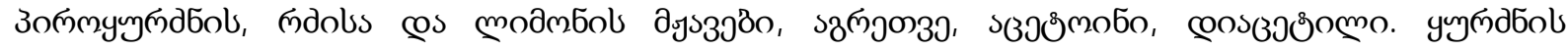

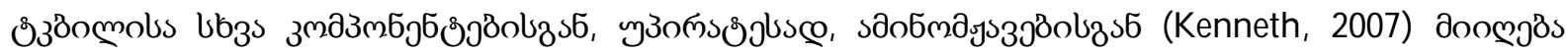

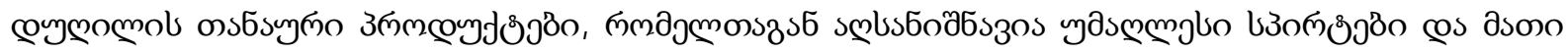

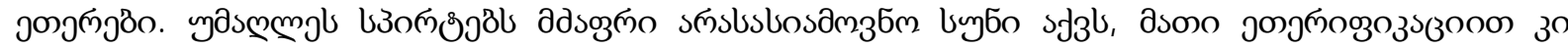

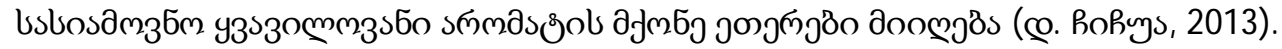

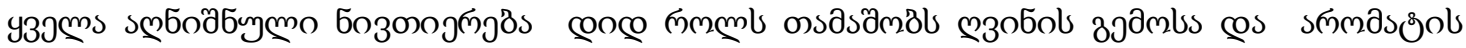

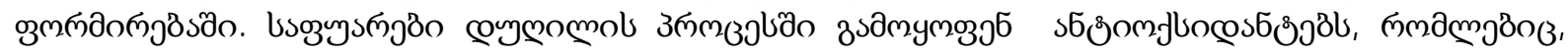

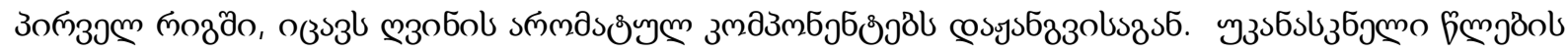

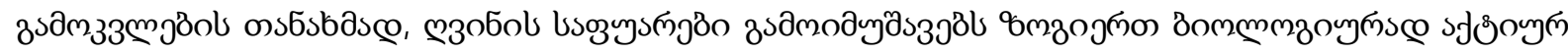

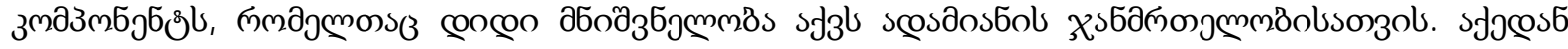

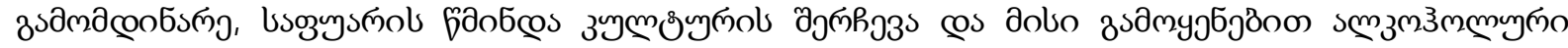

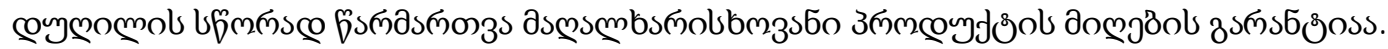

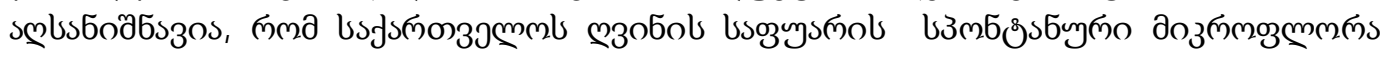

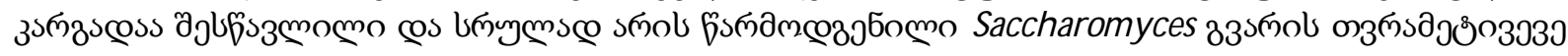

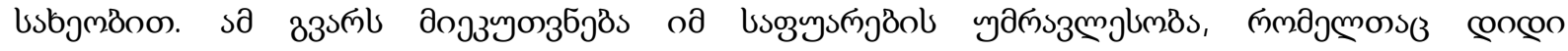

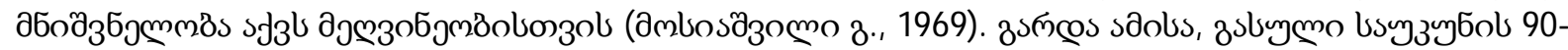

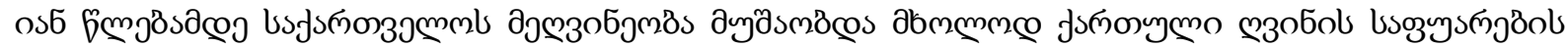




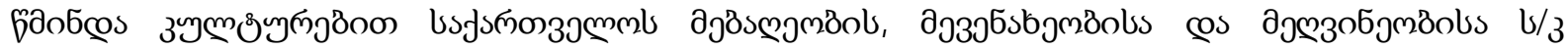

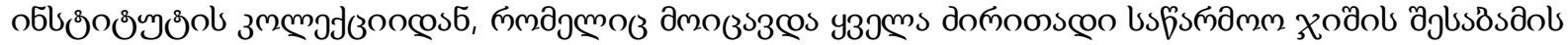

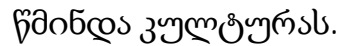

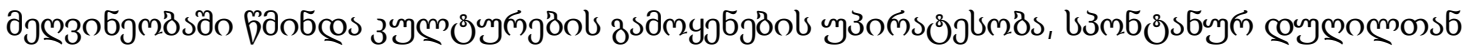

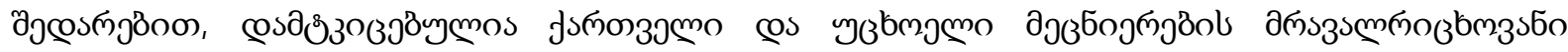

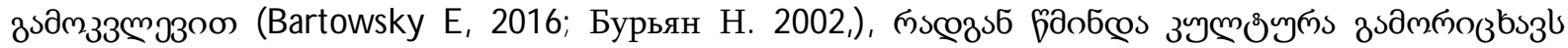

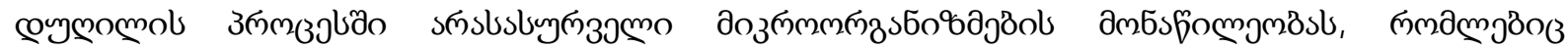

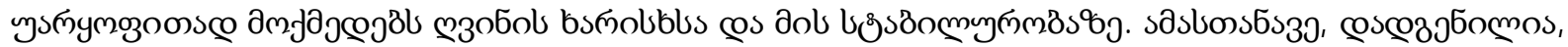

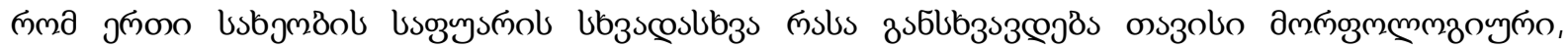

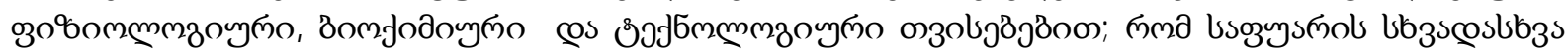

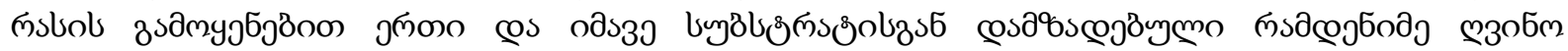

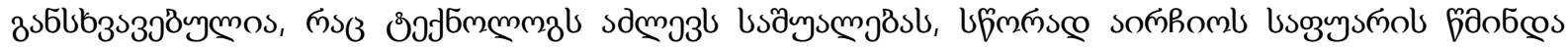

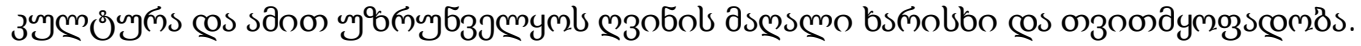

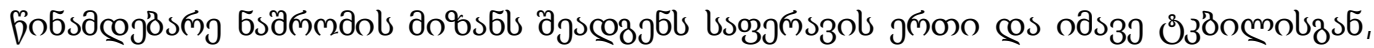

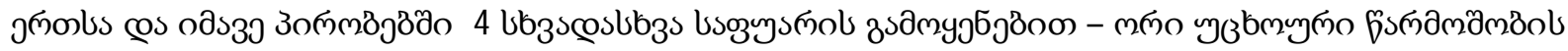

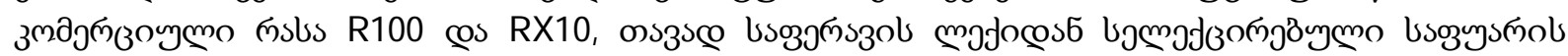

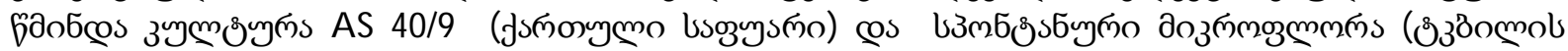

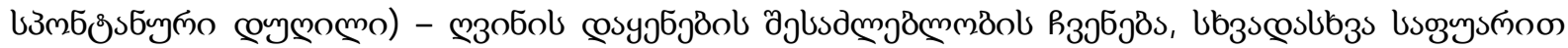

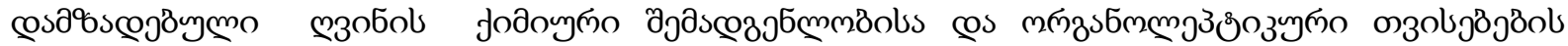

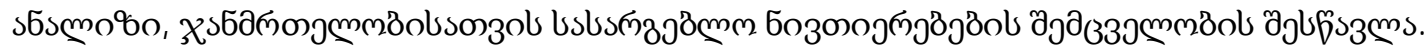

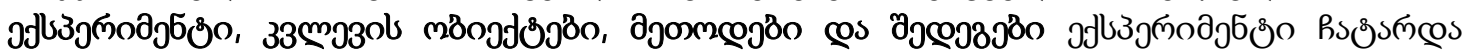

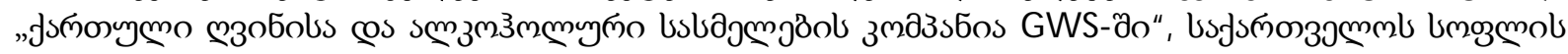

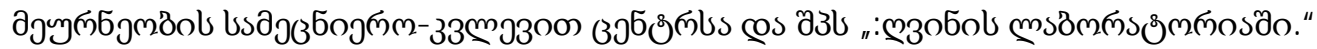

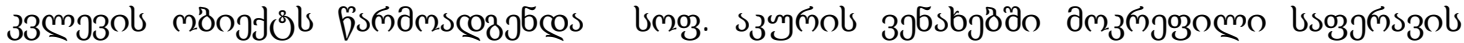

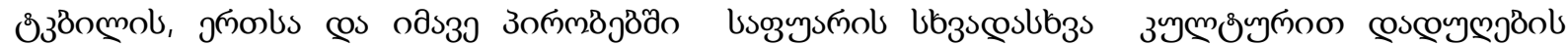

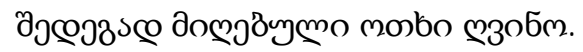

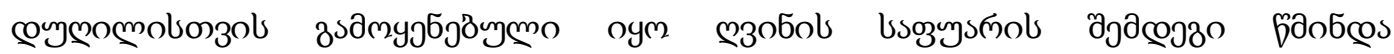

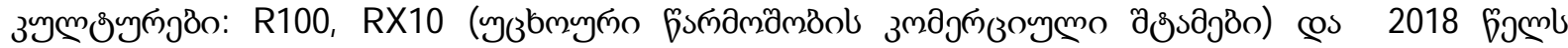

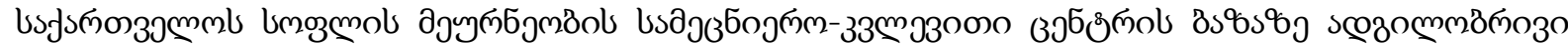

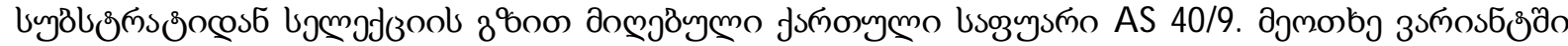

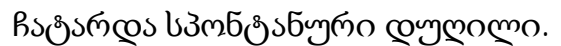

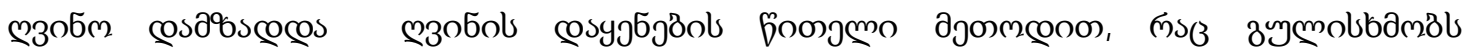

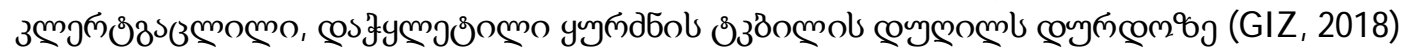

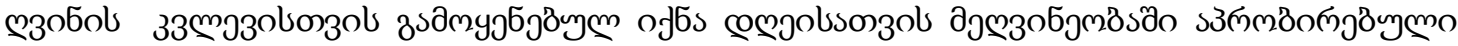

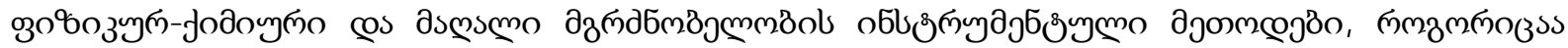

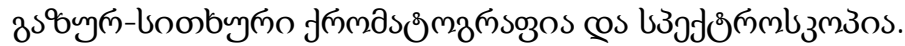

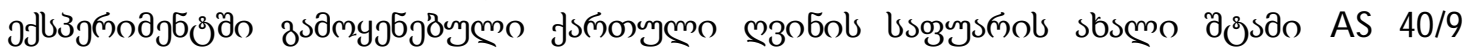

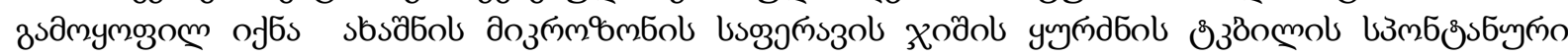

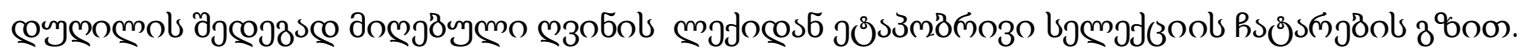

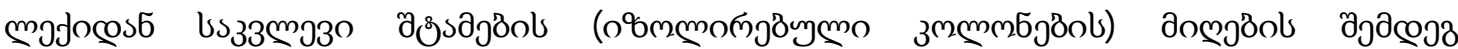

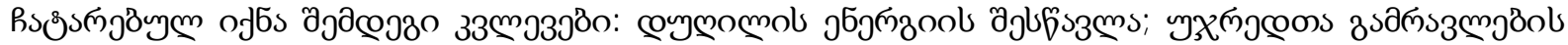

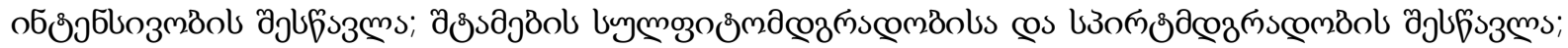

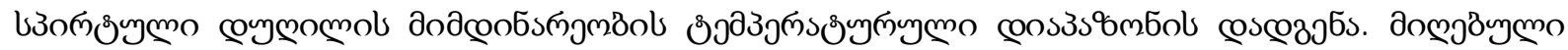
дащ

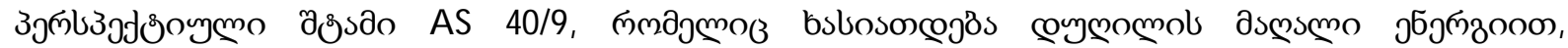

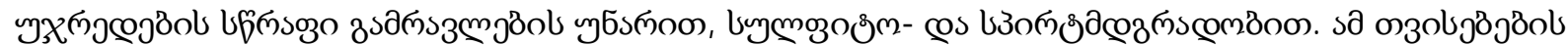

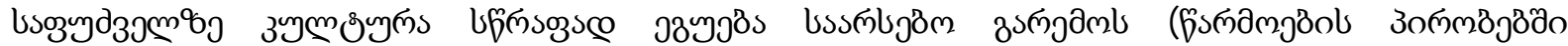

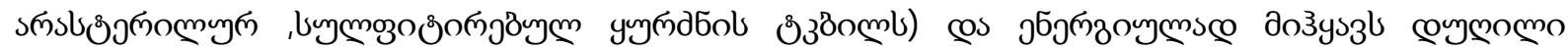

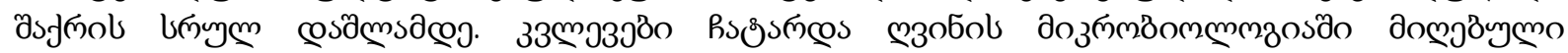

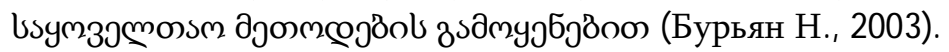




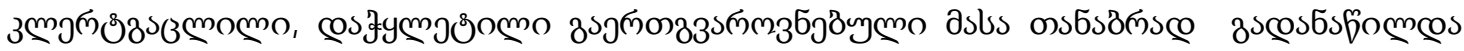

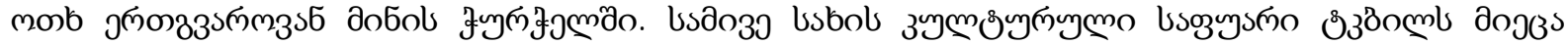

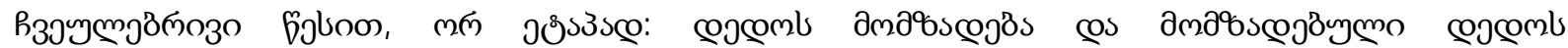

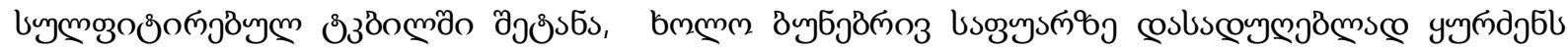

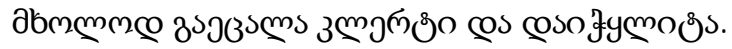

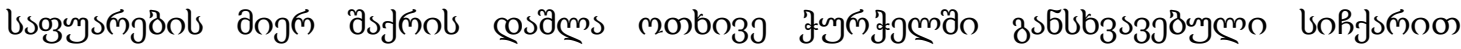

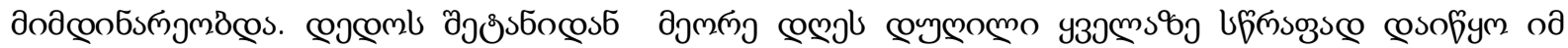

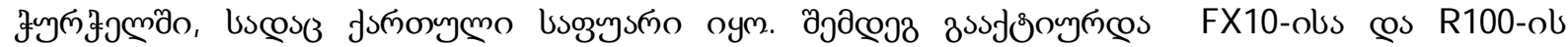

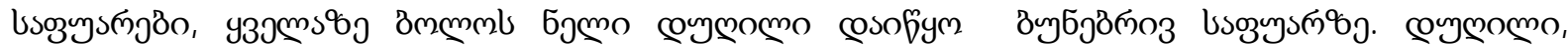

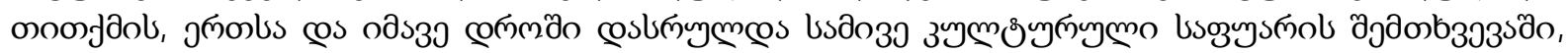

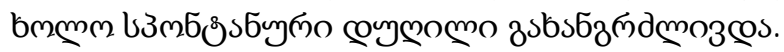

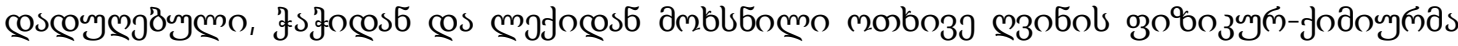

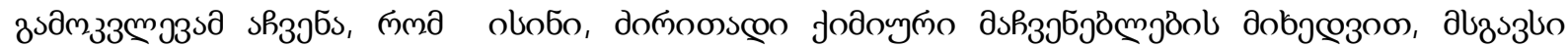

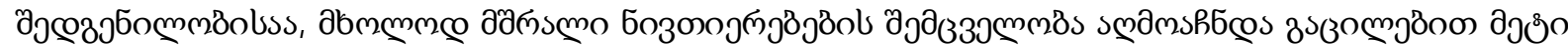

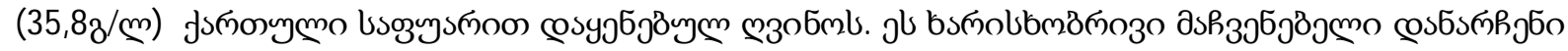

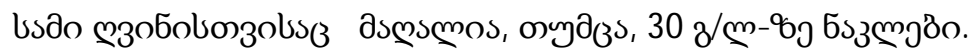

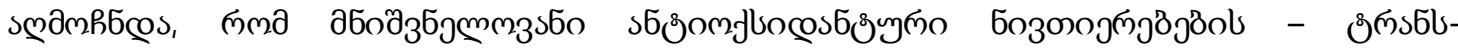

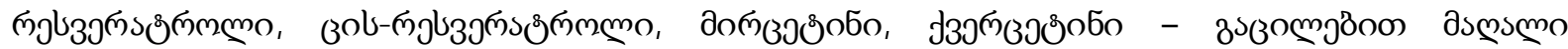
дsһ

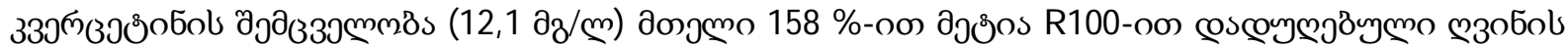

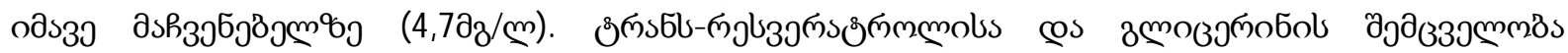

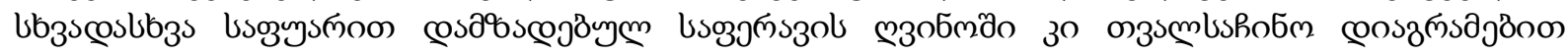

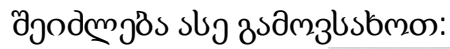

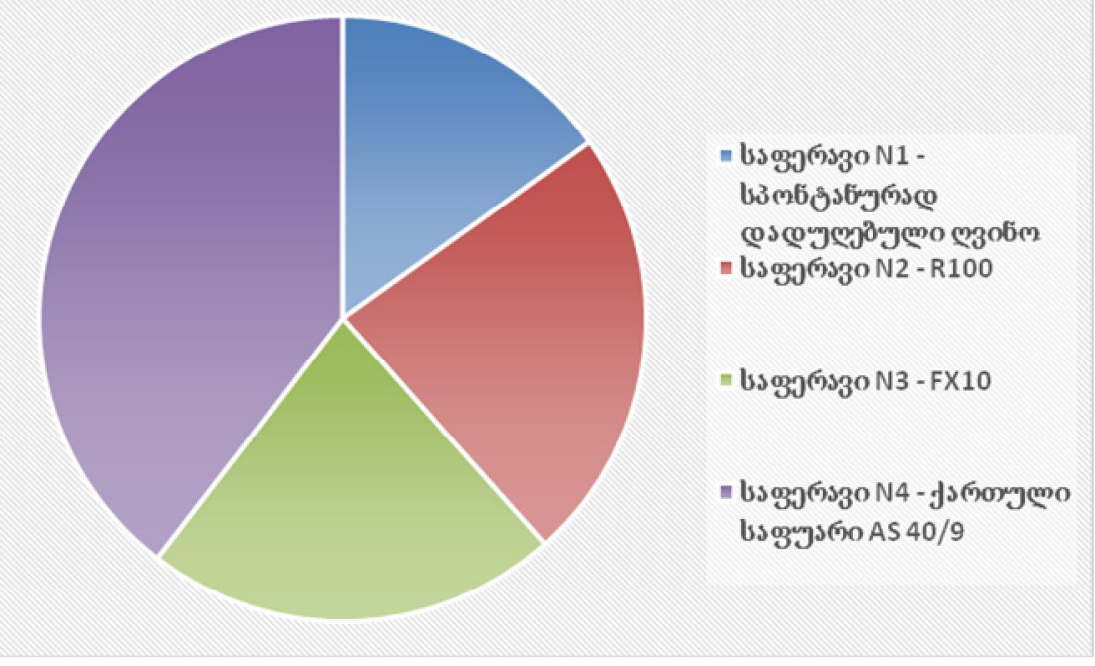




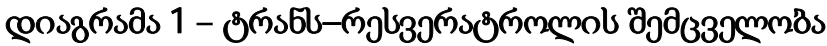

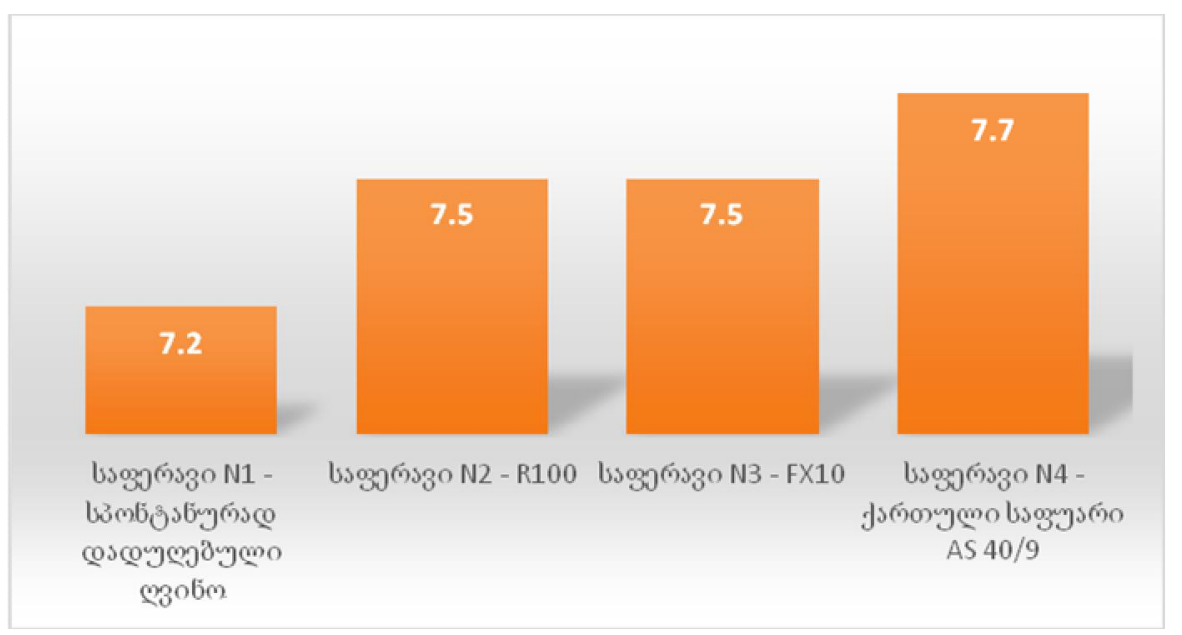

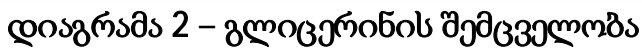

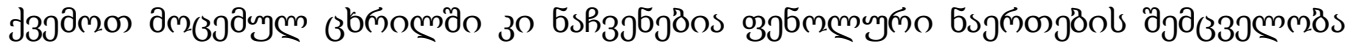

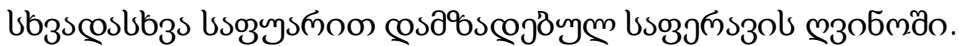

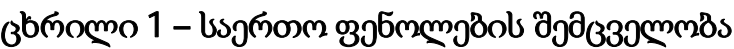

\begin{tabular}{|c|c|}
\hline Бодỹob cosbsbjmgßs & 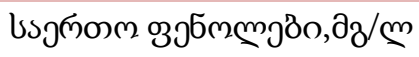 \\
\hline 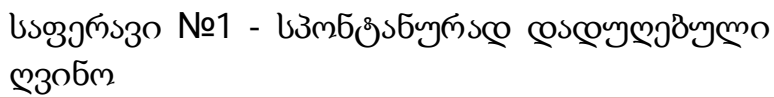 & 2158 \\
\hline 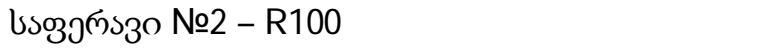 & 2148 \\
\hline 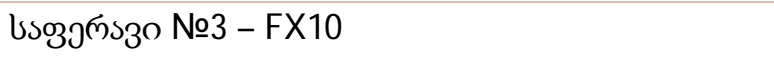 & 2222 \\
\hline 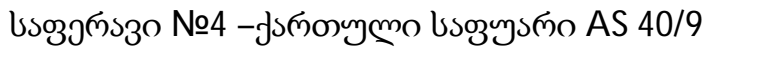 & 2355 \\
\hline
\end{tabular}

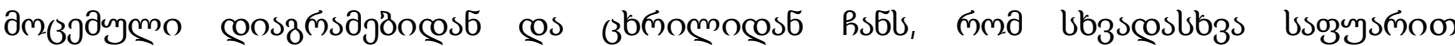

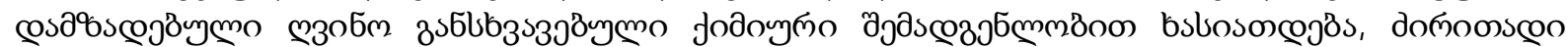

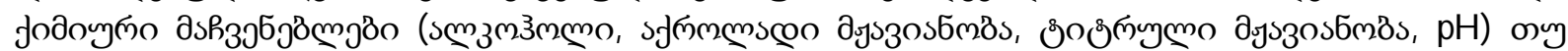

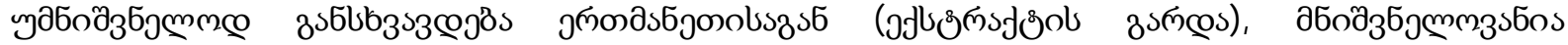

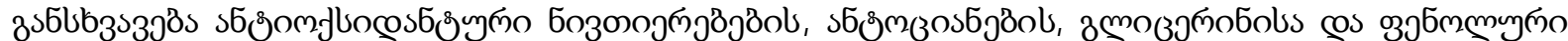

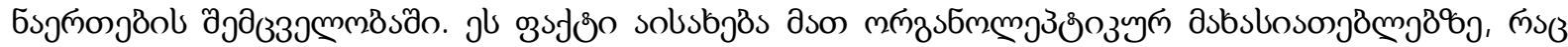

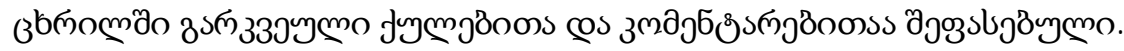

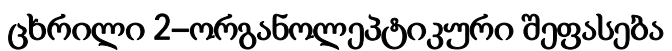

\begin{tabular}{|c|c|c|}
\hline 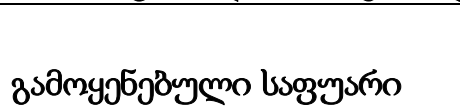 & 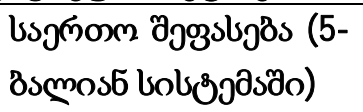 & змајбరతి \\
\hline $\begin{array}{l}\text { jుஙnomymo bsozysmo AS } \\
40 / 9\end{array}$ & 4,3 & 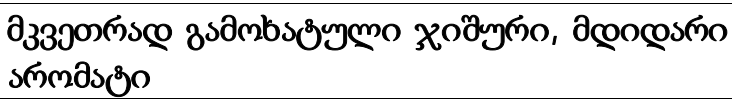 \\
\hline FX10 & 4.4 & 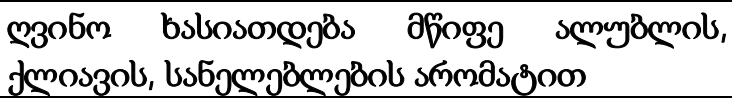 \\
\hline R100 & 4.1 & 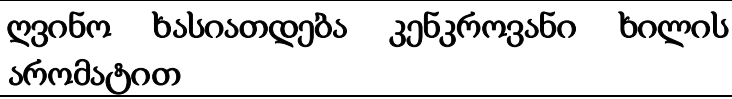 \\
\hline 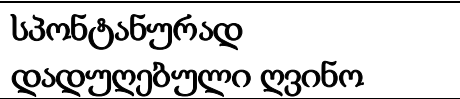 & 3.5 & 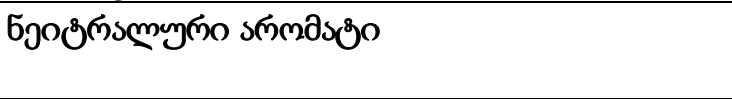 \\
\hline
\end{tabular}




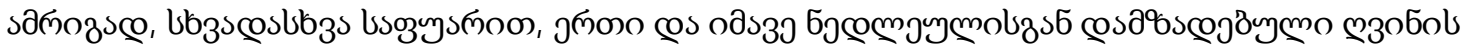

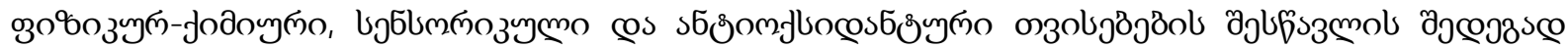

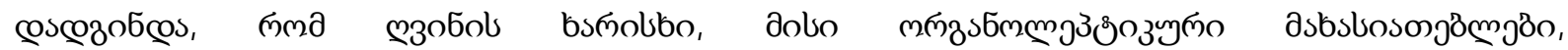

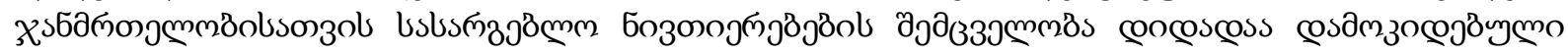

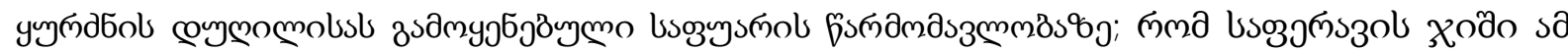

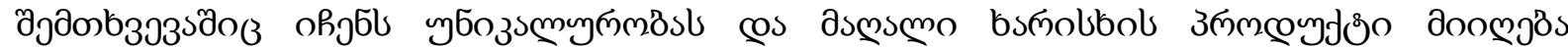

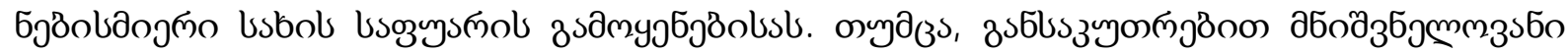

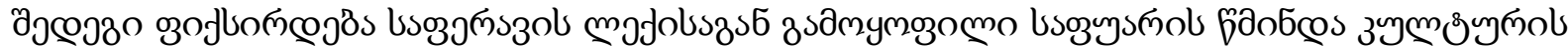

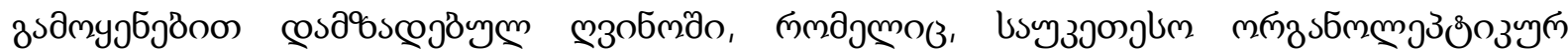

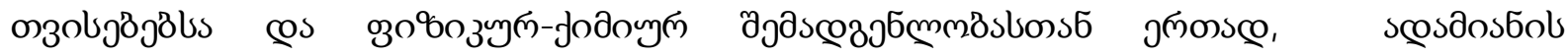

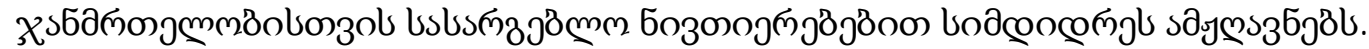

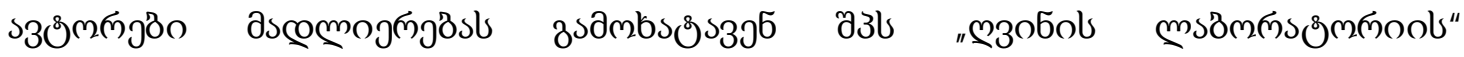

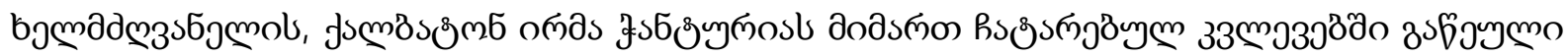

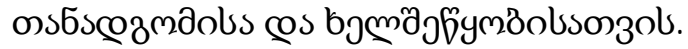

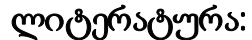

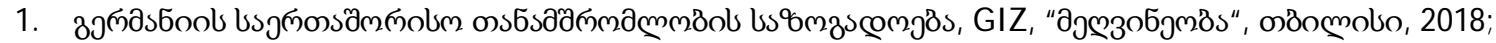

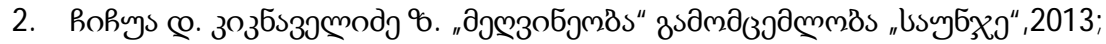

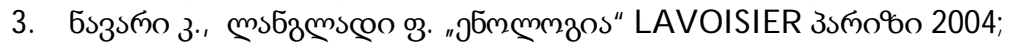

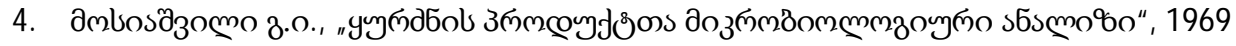

5. Ribereau-Gay on $P$,, Dubourdieu D. "The Microbiology of W ine and Vinifications $2^{\text {nd }}$ Edition" Copyright 2006 John W iley \& Sons Ltd, The A trium, Southern Gate, Chichester, W est Sussex P019 8SQ, England;

6. Kenneth C. Fugelsang, Charles G. Edwards "W ine M icrobiology" Second edition, "Springer" 2007;

7. Bartowsky E., Microbiology of winemaking, 2016;

8. Бурьян Н. И., Микробиология виноделия, Ялта.2002, 430с.

9. Бурьян Н. И., Практическая микробиология виноделия , Симферополь: Таврида, 2003

10. http://gwa.ge/letters/s/.

\section{Effect of Pure Culture of Yeast on the Characteristics of Wine Quality}

\author{
Zakaidze Ketevan \\ Iakob Gogebashvili State University, Telavi

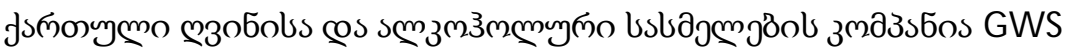 \\ Salia Elene \\ Georgian Scientific - Research Center of Agriculture,

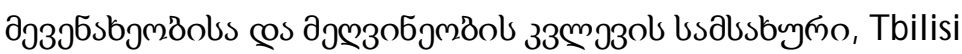 \\ Vephkhishvili Nino \\ Iakob Gogebashvili State University, Telavi
}

\begin{abstract}
Saperavi is one of the most distinguished varieties of wine among the grape varieties widely spread in Georgia. Along with the grapevine variety, the pure culture of yeast also plays a crucial role in producing high quality wine material, because wine is the product of the viability of yeast. All chemical transformations that take place during the alcoholic fermentation, as a result of which the grape juice is turned into a qualitatively new product, i.e. wine, are carried out by direct involvement of the ferment system of the yeast. In addition, in the process of viability, the yeast produces not only the primary products of fermentation (ethanol and carbon dioxide), but also a
\end{abstract}


number of organic substances (secondary and by -products) that play a big role in forming the wine taste and aroma, as well as the antioxidants, which provide its sustainability, and some biologically active components. Therefore, selecting the pure culture of wine yeast and directing the alcoholic fermentation correctly with the use of it, will guarantee to obtain a high quality product.

The paper presents the production of wine materials from the same must of the grape variety Saperavi, using different cultures of yeast (two foreign commercial cultures R100 and RX10, Georgian wine yeast type AS40/9 picked out through spontaneous microflora and selection) under the same conditions, it also provides the study of their chemical composition and organoleptic characteristics.

The experiment was conducted in "Georgian Wines and Spirits Company GWS," the Georgian Scientific - Research Center of Agriculture and the "Wine Laboratory" Ltd.

The experiment showed that the quality of each wine, its organoleptic characteristics, content of nutrients useful for health depend on the origin of the pure culture of the yeast applied; it should be noted that all the four versions produced high quality product, however, especially significant results were revealed in the wine produced using the pure culture of Georgian wine yeast, in particular:

- $\quad$ Content of dry matter is highest (extract);

- $\quad$ Aroma of grape variety is most distinct;

- $\quad$ Distinguished by the content of antioxidant substances, such as Trans- resveratrol, Cis Resveratrol, Myricetin and Quercetin.

- $\quad$ The wine made with Georgian yeast contains a high content of total phenolic compounds and anthocyanins as well as glycerin.

Key words: Saperavi, yeast, antioxidant substances, phenolic compounds, Trans - resveratrol

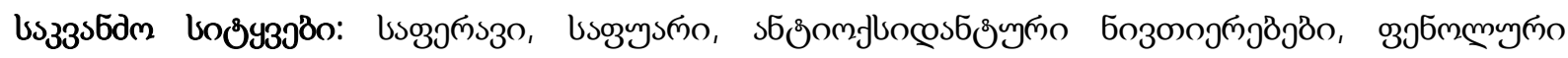

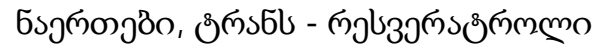

